\title{
Gating the Polarity of Endocannabinoid-Mediated Synaptic Plasticity by Nitric Oxide in the Spinal Locomotor Network
}

\author{
Jianren Song, Alexandros Kyriakatos, and Abdeljabbar El Manira \\ Department of Neuroscience, Karolinska Institutet, 17777 Stockholm, Sweden
}

The final motor output underlying behavior arises from an appropriate balance between excitation and inhibition within neural networks. Retrograde signaling by endocannabinoids adapts synaptic strengths and the global activity of neural networks. In the spinal cord, endocannabinoids are mobilized postsynaptically from network neurons and act retrogradely on presynaptic cannabinoid receptors to potentiate the locomotor frequency. However, it is still unclear whether mechanisms exist within the locomotor networks that determine the sign of the modulation by cannabinoid receptors to differentially regulate excitation and inhibition. In this study, using the lamprey spinal cord in vitro, we first report that 2-AG (2-arachidonyl glycerol) is mobilized by network neurons and underlies a form of modulation that is embedded within the locomotor networks. We then show that the polarity of the endocannabinoid modulation is gated by nitric oxide to enable simultaneously potentiation of excitation and depression of inhibition within the spinal locomotor networks. Our results suggest that endocannabinoid and nitric oxide systems interact to mediate inversion of the polarity of synaptic plasticity within the locomotor networks. Thus, endocannabinoid and nitric oxide shift in the excitation-inhibition balance to set the excitability of the spinal locomotor network.

\section{Introduction}

Neural networks underlying behavior undergo continuous plasticity that adapts their output contingent on previous activity. In networks with relatively fixed connectivity, modulatory systems produce adaptive changes by adjusting neuronal properties and synaptic strengths (Nusbaum et al., 2001; Abbott and Regehr, 2004; Destexhe and Marder, 2004; Kristan et al., 2005; LeBeau et al., 2005; Toledo-Rodriguez et al., 2005; Harris-Warrick, 2011). The resulting shift in the net excitability of the network mostly involves activation of distinct transmitter receptors that differentially affect excitatory and inhibitory synaptic transmission (e.g., dopamine) (Albin et al., 1989; Kreitzer and Malenka, 2007; Surmeier et al., 2007). However, it is still unclear whether mechanisms exist that reverse the polarity of the modulation by a single receptor to differentially regulate excitation and inhibition.

The spinal cord contains networks of neurons [central pattern generator (CPG)] sufficient and necessary to produce coordinated locomotor movements (Grillner, 1975; Kiehn, 2006; Goulding, 2009; Grillner and Jessell, 2009; Fetcho and McLean, 2010; Roberts et al., 2010; Büschges et al., 2011). The level of excitability of the spinal locomotor networks has to be tightly controlled to allow for movements of variable speed and intensity. This is often achieved by in-

Received Nov. 23, 2011; revised Jan. 30, 2012; accepted Feb. 19, 2012.

Author contributions: J.S., A.K., and A.E.M. designed research; J.S. and A.K. performed research; J.S., A.K., and A.E.M. analyzed data; J.S., A.K., and A.E.M. wrote the paper.

This work was supported by a grant from the Swedish Research Council, European Commission (FP7, Spinal Cord Repair), and Karolinska Institutet. We thank Drs. J. Ausborn, R. Hill, and S. Grillner for comments and critical discussion of this manuscript.

Correspondence should be addressed to Abdeljabbar El Manira, Department of Neuroscience, Karolinska Institutet, 17177 Stockholm, Sweden. E-mail: abdel.elmanira@ki.se.

A. Kyriakatos's present address: Laboratory of Sensory Processing, Brain Mind Institute, Faculty of Life Sciences, École Polytechnique Fédérale de Lausame (EPFL), Switzerland.

DOI:10.1523/JNEUROSCI.5850-11.2012

Copyright $\odot 2012$ the authors $\quad 0270-6474 / 12 / 325097-09 \$ 15.00 / 0$ volving modulatory systems (El Manira et al., 2008; Sillar et al., 2008; El Manira and Kyriakatos, 2010; Harris-Warrick, 2011; Jordan and Slawinska, 2011; Miles and Sillar, 2011). In the lamprey spinal cord, endocannabinoids are released from CPG neurons and contribute to setting the baseline locomotor frequency (Kettunen et al., 2005; Kyriakatos and El Manira, 2007). Two main endocannabinoids have been identified, 2-arachidonyl glycerol (2-AG) and anandamide, which are synthesized and degraded by separate pathways (Sugiura et al., 2002; Piomelli, 2003; Di Marzo, 2008). They are released from postsynaptic neurons and act retrogradely on presynaptic type 1 cannabinoid receptors $\left(\mathrm{CB}_{1} \mathrm{Rs}\right)$ to match transmitter release to the level of postsynaptic excitability (Kim et al., 2002; Freund et al., 2003; Chevaleyre et al., 2006; Mackie and Stella, 2006; Katona and Freund, 2008; Kano et al., 2009; El Manira and Kyriakatos, 2010).

In many CNS regions, $\mathrm{CB}_{1}$ Rs activation by $2-\mathrm{AG}$ has been shown to exclusively induce synaptic depression and in no case has synaptic potentiation been directly linked to direct activation of this receptor (Alger, 2002; Freund et al., 2003; Chevaleyre et al., 2006; Katona and Freund, 2008; Lovinger, 2008; Heifets and Castillo, 2009; Kano et al., 2009) (but see Cachope et al., 2007). In this study, we first show that 2-AG is released in the locomotor network. Blocking its synthesis or degradation affected the locomotor rhythm in a manner consistent with a role in potentiating the frequency by shifting the balance between excitation and inhibition. We then show that the inversion of the polarity of the modulation of excitatory and inhibitory synaptic transmission by the endocannabinoid 2-AG is gated by nitric oxide (NO). Scavenging NO or inhibiting its synthesis blocked the potentiation of excitation, but not the depression of inhibition. Our results thus indicate that $\mathrm{NO}$ gates the sign of $\mathrm{CB}_{1}$ Rs modulation of synaptic transmission by 2-AG within the locomotor CPG to potentiate excitation and the locomotor output. 


\section{Materials and Methods}

All experiments were performed on in vitro preparations of the isolated, intact spinal cord from adult lampreys (Lampetra fluviatilis) of either sex. All protocols were approved by the Animal Research Ethical Committee, Stockholm. Lampreys were anesthetized with MS-222 (100 mg/L; Sigma-Aldrich) and eviscerated, and the lateral muscle walls were removed. The spinal cord and notochord were dissected and pinned in a cooled $\left(8-12^{\circ} \mathrm{C}\right)$ Sylgard-lined experimental chamber continuously perfused with physiological solution. The control solution was composed of the following (in mM): $138 \mathrm{NaCl}, 2.1 \mathrm{KCl}, 1.8 \mathrm{CaCl}_{2}, 1.2$ $\mathrm{MgCl}_{2}, 4$ glucose, and 2 HEPES, bubbled with $\mathrm{O}_{2}$ and $\mathrm{pH}$ adjusted to 7.4. Fictive swimming activity was induced by adding $50-100 \mu \mathrm{M}$ NMDA to the physiological solution. Alternating locomotor burst activity was recorded by en passant glass suction electrodes placed on two opposing ventral roots at their exits from the spinal cord. After perfusion of NMDA, the locomotor burst frequency increased gradually over time and reached a stable level after 3-4 h. Pharmacological reagents were normally added only after the burst frequency had been stable for at least $1 \mathrm{~h}$. Two minute recordings of ventral root activity were sampled every $5 \mathrm{~min}$. The cycle duration was measured and averaged from 100 to 200 consecutive cycles. The cycle duration was defined as the time interval between the onsets of two consecutive bursts, and the burst frequency was calculated as the inverse of the averaged cycle duration. To examine the changes in midcycle reciprocal inhibition during locomotion, intracellular recordings were made from motoneurons (MNs) and rhythmically active unidentified interneurons with $3 \mathrm{~m}$ potassium acetate-filled thin-walled glass microelectrodes with a resistance of $15-30 \mathrm{M} \Omega$. MNs were identified by recording their axonal action potentials in a one-to-one manner from the corresponding ventral root through the extracellular suction electrode. All MNs recorded during fictive locomotion received phasic excitation alternating with phasic inhibition. The concurrent recordings of the locomotor frequency and of the activity of MNs allowed us to correlate changes in the synaptic amplitude with changes in locomotor frequency. Two minute recordings of intracellular and extracellular activity were sampled every $5 \mathrm{~min}$. The spikes were removed using a digital low-pass filter $(30 \mathrm{~Hz})$, and the synaptic input was averaged from 100 to 200 cycles; their duration was normalized and aligned according to the membrane potential of the recorded neurons. The peak-to-trough amplitude of the locomotorrelated synaptic input was measured and monitored in control and in the presence of different pharmacological agents.

To examine the changes in ipsilateral excitatory or reciprocal inhibitory synaptic transmission, the recording chamber was divided into two pools by an agar barrier (Dale, 1986). Locomotor activity was induced in the rostral pool by NMDA while the spinal cord in the caudal pool was perfused with physiological solution containing strychnine to block inhibitory synaptic transmission or kynurenic acid to block ionotropic glutamate receptors. After $30 \mathrm{~min}$ in strychnine or kynurenic acid, intracellular recordings were made from MNs two to three segments away from the agar barrier. In the presence of strychnine, excitatory synaptic input occurred in phase with the activity of the ipsilateral ventral root in the rostral pool. Inhibitory synaptic input was recorded in the presence of kynurenic acid and occurred in phase with the contralateral ventral root. Two minute recordings of intracellular and extracellular activities were sampled every $5 \mathrm{~min}$, and the amplitude of the excitatory or inhibitory drive and the frequency of the locomotor bursts were monitored in control and in the presence of different pharmacological agents. In experi-
B NMDA + 2-AG

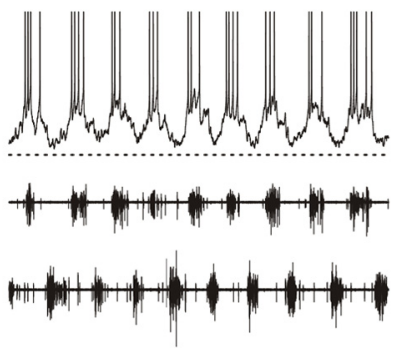

D $2-A G$

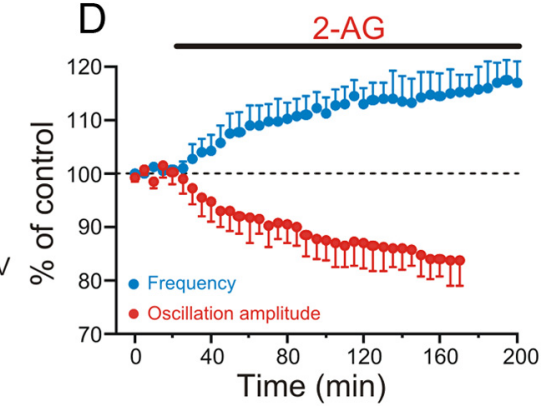

NMDA

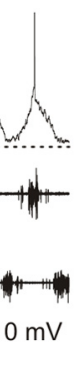

$1 \mathrm{~s}$

NMDA + 2-AG

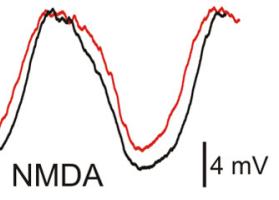

$(\min )$

Figure 1. Increase in the locomotor burst frequency by 2-AG. A, Locomotor rhythm was induced by NMDA and recorded in opposing ventral roots. Intracellular recording from a MN that received on-cycle excitation in phase with the ipsilateral ventral root (vr-i) activity that filtered out using a low-pass filter and normalized. $\boldsymbol{D}$, Averaged data from all the experiments showing the time course of decrease of the oscillation amplitude (red), which is paralleled with an increase in the locomotor burst frequency (blue) induced by 2-AG.

ments in which the NO scavenger 2-(4-carboxyphenyl)-4,4,5,5tetramethylimidazoline-1-oxyl-3-oxide (carboxy-PTIO) was tested, it was first applied for $1 \mathrm{~h}$ before application of 2-AG either to the entire spinal cord or in the caudal pool in experiments with split-bath configuration. The effects of $2-\mathrm{AG}$ in preparations with preincubation with carboxy-PTIO were compared with those obtained in separate control preparations without carboxy-PTIO preincubation.

Intracellular current-clamp recordings were made in bridge mode with an Axoclamp 2B amplifier (Molecular Devices). pClamp software (Molecular Devices) was used for data acquisition and analysis on a personal computer equipped with an analog/digital interface (Digidata 1300). Recordings were also acquired and stored digitally using pClamp. Data analysis of burst frequency and synaptic transmission was done off-line using the program Spike2 (Cambridge Electronic Design). The values reported correspond to mean \pm SEM, and $n$ represents the number of experiments. The statistical significance was assessed with paired or unpaired Student's $t$ tests, and differences were considered significant if $p<0.05$.

The following drugs were used in this study: NMDA (100 $\mu$; Tocris), strychnine (5 $\mu \mathrm{M}$; Sigma-Aldrich), kynurenic acid (2 mm; Sigma-Aldrich), 2-AG (1-2 $\mu \mathrm{M}$; Tocris), the NO scavenger carboxy-PTIO (150 $\mu \mathrm{M}$; Tocris), tetrahydrolipstatin (THL) (10-20 $\mu \mathrm{m}$; Tocris), and 4-[bis(1,3-benzodioxol5-yl)hydroxymethyl]-1-piperidinecarboxylic acid 4-nitrophenyl ester (JZL184) (1-2 $\mu \mathrm{M}$; Tocris). The concentrations of THL and JZL184 used in this study were determined based on previously published work in mammals. All agonists and antagonists were dissolved as stock solutions in water except for 2-AG and JZL184, which were dissolved in DMSO. The same concentration of the solvent DMSO had no effect on the locomotor frequency and was always added to control solutions throughout the experiments.

\section{Results}

\section{Identity of the endocannabinoid in the spinal locomotor} circuit

Endocannabinoids are released within the spinal locomotor circuit in lamprey and contribute to setting the baseline of locomo- 
A

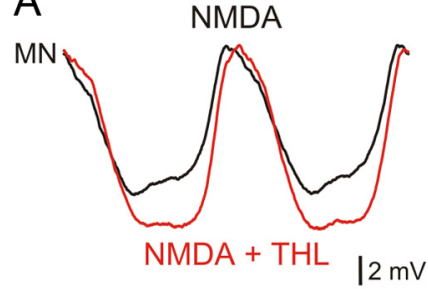

B

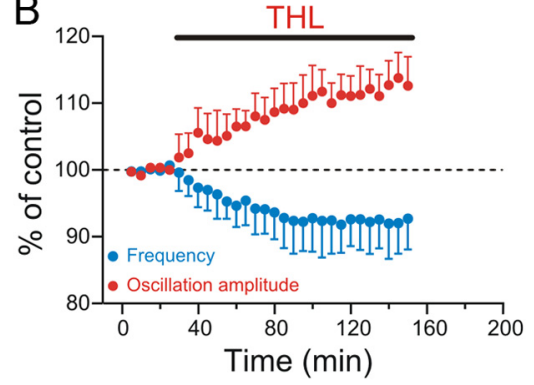

C

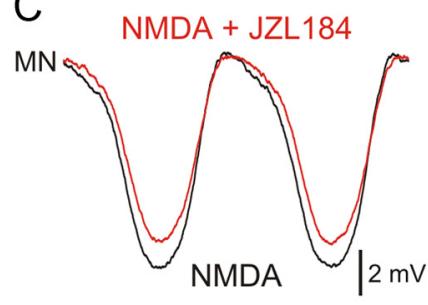

D

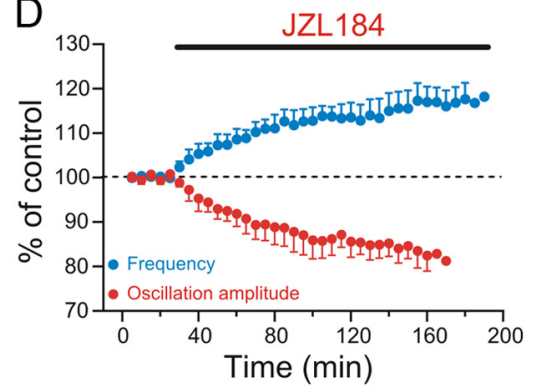

Figure 2. Identification of 2-AG as an endocannabinoid released within the locomotor network. $\boldsymbol{A}$, Averaged membrane potential oscillation displayed by a MN during locomotor activity. Inhibition of the 2-AG synthesizing enzyme DAG lipase by THL (10-20 $\mu \mathrm{M})$ increased the amplitude of the oscillation received by this MN during locomotor activity induced by NMDA. $\boldsymbol{B}$, Graph showing averaged data points over time in control and in the presence of THL. The increase in the oscillation amplitude (red) was accompanied with a decrease in the locomotor burst frequency (blue). $\boldsymbol{C}$, Inhibition of MGL, the degradation enzyme of 2-AG, by JZL184 (1-2 $\mu \mathrm{m}$ ) decreased the amplitude of the oscillation displayed by the recorded MN during locomotor activity induced by NMDA. $\boldsymbol{D}$, Graph showing averaged data points in control and in JZL184, which increased the locomotor burst frequency (blue) and decreased the oscillation amplitude (red).

tor frequency (Kettunen et al., 2005; Kyriakatos and El Manira, 2007). To determine the identity of the endocannabinoid responsible for this embedded modulation, we tested the effects of 2-AG and specific inhibitors of the enzymes involved in its synthesis and degradation. In our experiments, locomotor activity was induced by NMDA $(100 \mu \mathrm{M})$ and was monitored by recording the activity from two opposing ventral roots while membrane potential oscillations were recorded intracellularly from MNs (Fig. $1 A)$. Application of 2-AG $(1-2 \mu \mathrm{M})$ increased the locomotor burst frequency by $17.9 \pm 4.1 \%(p<0.001 ; n=6$; Fig. $1 A, B)$, which was accompanied by a concurrent decrease in the mean amplitude of the membrane potential oscillation to $83.7 \pm 4.7 \%$ comparing with control $(p<0.01 ; n=5$; Fig. $1 C, D)$ and an increase in the number of action potentials during the on-cycle depolarization from $1.1 \pm 0.1$ spike per cycle in control to $3.2 \pm$ 0.1 in $2-\mathrm{AG}(p<0.001 ; n=5$; Fig. $1 B)$. These results, which are in accord with previous findings (Kettunen et al., 2005; Kyriakatos and El Manira, 2007), show that exogenous application of 2-AG potentiates burst frequency and decreases the amplitude of the membrane potential oscillation during locomotion.

These results are consistent with the idea that 2-AG is released within the spinal circuit. To test this hypothesis, we interfered with 2-AG biosynthesis and degradation pathways by using specific inhibitors. 2-AG is liberated from diacylglycerol (DAG) by DAG lipase (DGL) and is metabolized principally by monoglyceride lipase (MGL) in the nervous system. If 2-AG is released in the lamprey spinal cord, inhibition of DGL would impact the locomotor activity by decreasing the basal levels of 2-AG. Application of the specific DGL inhibitor THL (10-20 $\mu \mathrm{M}$ ) (Bisogno et al., 2003; Hashimotodani et al., 2008) decreased the locomotor burst frequency by $8.1 \pm 2.7 \%(p<0.01 ; n=6)$ and increased the amplitude of the membrane potential oscillation by $13.8 \pm$ $3.8 \%(p<0.01 ; n=5$; Fig. $2 A, B)$. Conversely, inhibition of $2-A G$ degradation should increase the basal levels of 2-AG and enhance its modulatory actions. Indeed, inhibition of MGL with the specific blocker JZL184 (1-2 $\mu \mathrm{M}$ ) (Pan et al., 2009) increased the locomotor burst frequency by $17.5 \pm 3.5 \%$ and decreased the amplitude of the membrane potential oscillation by $17.7 \pm 3.6 \%(p<$ 0.001; $n=6$; Fig. $2 C, D)$.

By interfering with the synthesis and degradation machinery, our results support the conclusion that 2-AG is released within the locomotor circuit. Furthermore, the data suggest that $2-\mathrm{AG}$ is continuously synthesized to help in setting the appropriate balance between inhibition and excitation from network neurons and thus regulates the baseline locomotor burst frequency.

\section{Endocannabinoids change the balance between excitation and inhibition}

The change in the synaptic drive received by network neurons during locomotion could be due to a shift in the balance between excitation and inhibition. We therefore tested how 2-AG and its endogenous mobilization affect synaptic transmission between network neurons during locomotion. In these experiments, the recording chamber was divided into two pools by an agar barrier, and locomotor activity was induced in the rostral pool with NMDA $(100 \mu \mathrm{M})$. The caudal pool was perfused with normal saline (without NMDA), and strychnine $(5 \mu \mathrm{M})$ was added to isolate the excitatory component of the rhythmic locomotor synaptic drive arising from interneurons whose axons project ipsilaterally across the agar barrier (Fig. $3 A$ ). In this configuration, the effect of endocannabinoids on the excitatory drive to motoneurons relevant for the generation of locomotor activity can be monitored (Fig. 3B). Application of 2-AG in the caudal pool increased the amplitude of the excitatory synaptic drive in the recorded motoneurons to $113.9 \pm 3.3 \%$ of control values $(p<$ $0.01 ; n=6$; Fig. $3 C-E$ ).

The above results show that activation of cannabinoid receptors by exogenous application of $2-A G$ potentiates excitatory synaptic transmission within the locomotor network. The question that arises is whether there is a tonic modulation of excitatory synaptic transmission by endocannabinoids released within the spinal cord that contributes to the regulation of the burst frequency during locomotion. To answer this question, inhibitors of the enzymes responsible for 2-AG biosynthesis and degradation were used. Inhibition of the DAG lipase with THL $(10-20 \mu \mathrm{M})$ decreased the amplitude of the locomotor-related excitatory synaptic drive to motoneurons by $16.3 \pm 2.2 \%(p<0.001 ; n=7$; Fig. $3 F, G)$. However, inhibition of the $2-\mathrm{AG}$ degradation enzyme MGL with JZL184 (1-2 $\mu \mathrm{M})$ increased the amplitude of the excitatory drive received by $13.1 \pm 3.3 \%$ on motoneurons during locomotion $(p<0.001 ; n=6$; Fig. $3 H, I)$. In these experiments, 2-AG, THL, and JZL184 had no effect on the locomotor burst frequency induced by NMDA in the rostral pool (Fig. $3 E, G, I$ ).

The potentiation of the excitatory drive could explain the increase in the firing of MNs and burst frequency during locomotion. However, it does not account for the overall decrease in the 
membrane potential oscillation amplitude (Fig. 1), which could involve a concomitant effect on inhibitory synaptic transmission. To test this possibility, experiments using the split-bath configuration with the caudal pool being perfused with normal saline containing kynurenic acid (2 mM) were performed to block excitatory synaptic transmission (Fig. 4A). Midcycle inhibitory synaptic transmission originating from active commissural interneurons in the rostral pool was monitored in motoneurons recorded in the caudal pool (Fig. 4B). Application of $2-A G$ in the caudal pool depressed the amplitude of the midcycle inhibition in motoneurons by $26.8 \pm 2.9 \%(p<0.001$; $n=7$; Fig. $4 C-E$ ). Conversely, blockade of the synthesizing enzyme DAG lipase with THL (10-20 $\mu \mathrm{M})$ increased the amplitude of the midcycle inhibition by $10.4 \pm 2.5 \%$ $(p<0.01 ; n=5$; Fig. $4 F, G)$, indicating that newly synthesized endocannabinoids are responsible for a tonic decrease of the midcycle inhibition during locomotion. If there is a tonic mobilization of the endocannabinoid 2-AG, the modulation of midcycle inhibition should be further enhanced by blockade of its degradation enzyme MGL. Indeed, application of the MGL inhibitor JZL184 decreased the amplitude of the midcycle inhibition by $15.8 \pm 2.6 \%(p<0.001 ; n=6$; Fig. $4 H, I)$. In these experiments, $2-\mathrm{AG}$, THL, and JZL184 had no effect on the locomotor burst frequency induced by NMDA in the rostral pool (Fig. 4E, G,I).

These results indicate that there is a tonic modulation by endocannabinoids of excitatory and inhibitory synaptic transmission from network neurons during locomotion. 2-AG is the endocannabinoid synthesized and mobilized within the locomotor network that tips the excitation-inhibition balance within the locomotor network in favor of excitation by differentially potentiating excitatory and depressing inhibitory synaptic transmission and thereby synergistically increasing the locomotor burst frequency.

\section{Inversion of the polarity of endocannabinoid modulation by nitric oxide}

The endocannabinoid 2-AG appears to modulate excitatory and inhibitory synaptic transmission within the locomotor network in opposite directions. While the depression of synaptic transmission has been reported previously, potentiation of excitation is novel. What mechanism is responsible for the inversion of the polarity of the endocannabinoid modulation of excitation and inhibition? We previously
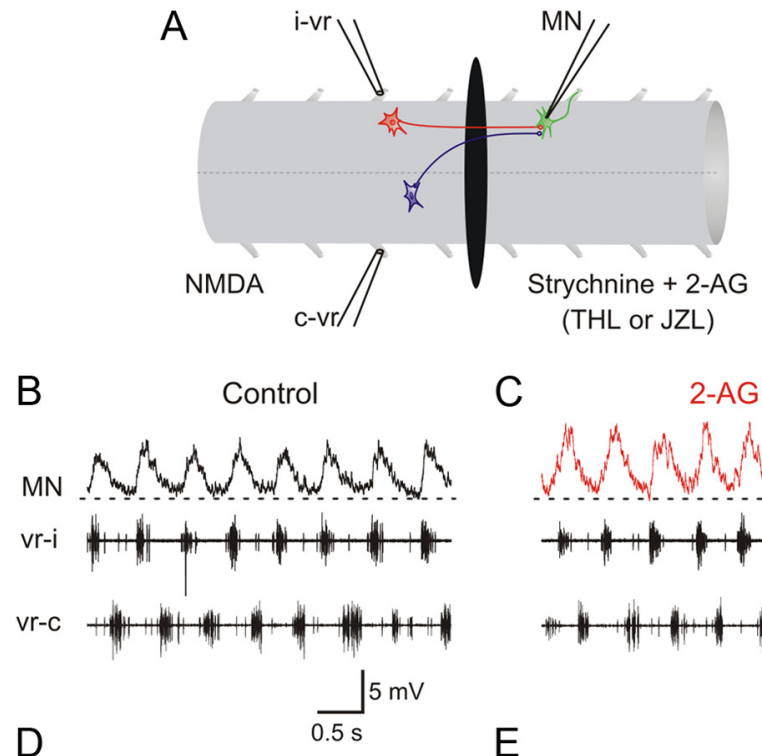

C 2-AG
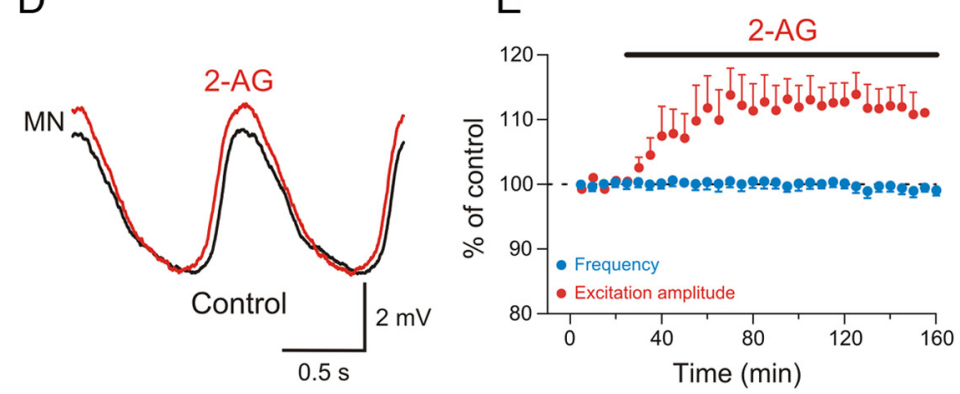

F
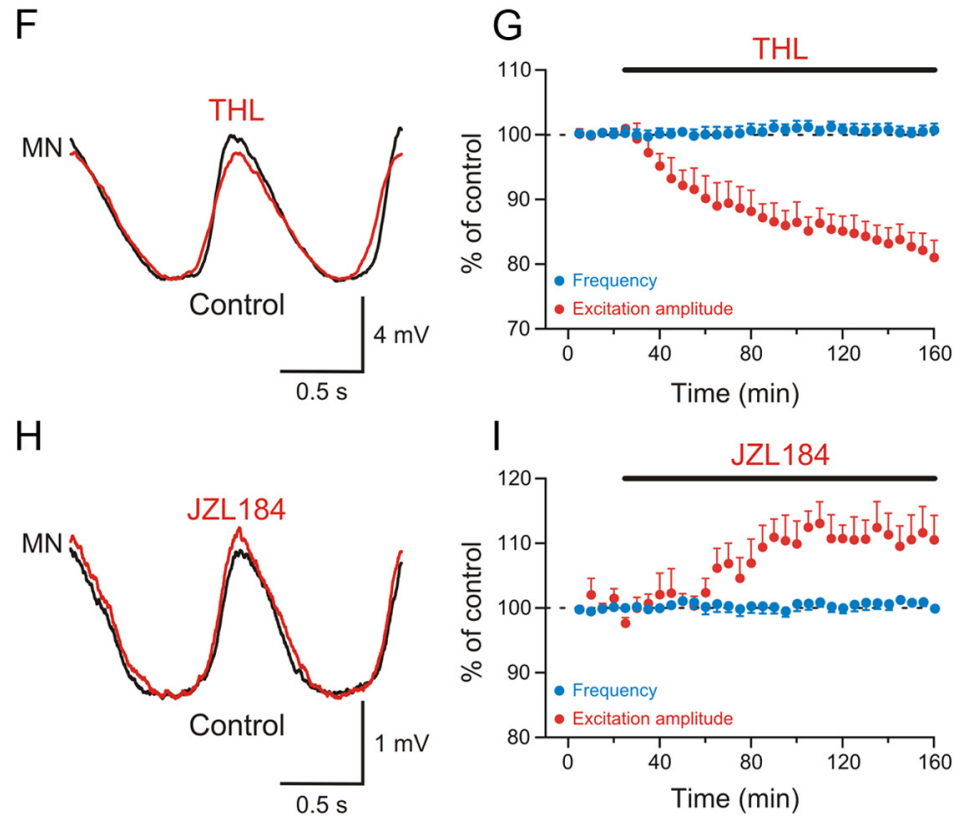

Figure 3. Potentiation of excitatory synaptic transmission by the endocannabinoid 2-AG. $A$, The recording chamber was divided into two pools with an agar barrier, and locomotor activity was induced by NMDA in the rostral part while locomotor-driven excitation was recorded in a MN in the caudal pool, which was perfused with normal saline solution containing strychnine to block inhibition. $\boldsymbol{B}$, The recorded MN received excitatory inputs occurring in phase with the ipsilateral ventral root burst. C, Application of 2-AG to the caudal pool increased the amplitude of excitatory synaptic input received by the MN. D. Averaged excitatory synaptic input received by the MN showing an increase in amplitude induced by $2-A G$. $E$, Plot showing average data from different experiments showing the time course of the change in the amplitude of excitation in the caudal pool (red) and the locomotor burst frequency in the rostral pool (blue) before and during 2-AG application. $\boldsymbol{F}$, Average excitatory synaptic input showing a decrease in amplitude induced by THL. $\boldsymbol{G}$, Plot of averaged data from different experiments showing the time course of the change in the amplitude of the excitation in the caudal pool (red) and the locomotor burst frequency in the rostral pool (blue) in control and in THL. $\boldsymbol{H}$, Application of JZL184 in the caudal pool increased the amplitude of the excitatory input received by the MN. I, Plot of averaged data from different experiments showing the change in the amplitude of excitation in the caudal pool (red) and the locomotor burst frequency in the rostral pool (blue) in control and in JZL184. 


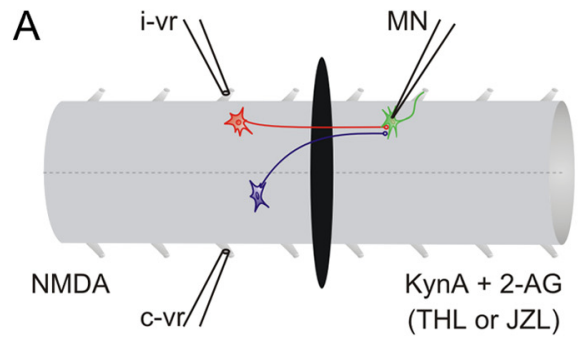

B

Control

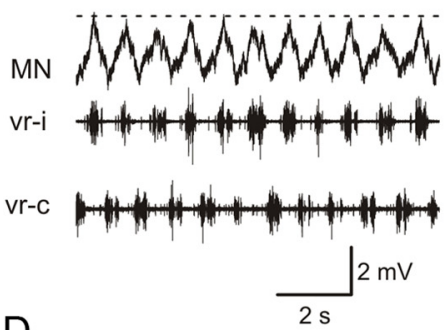

C
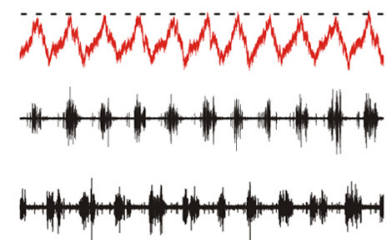

$\mathrm{E}$
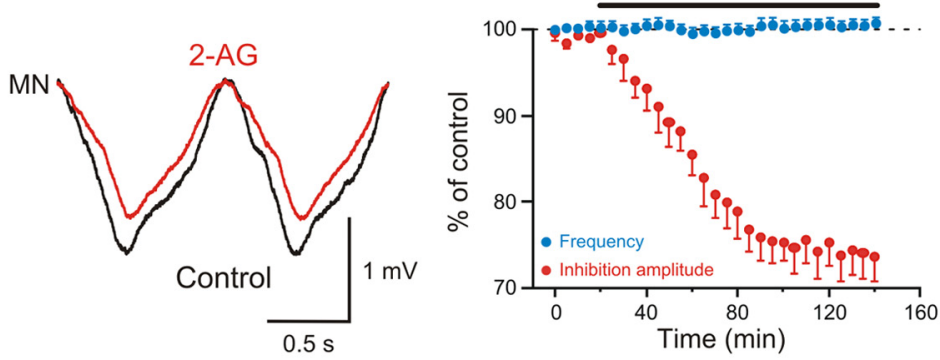

F
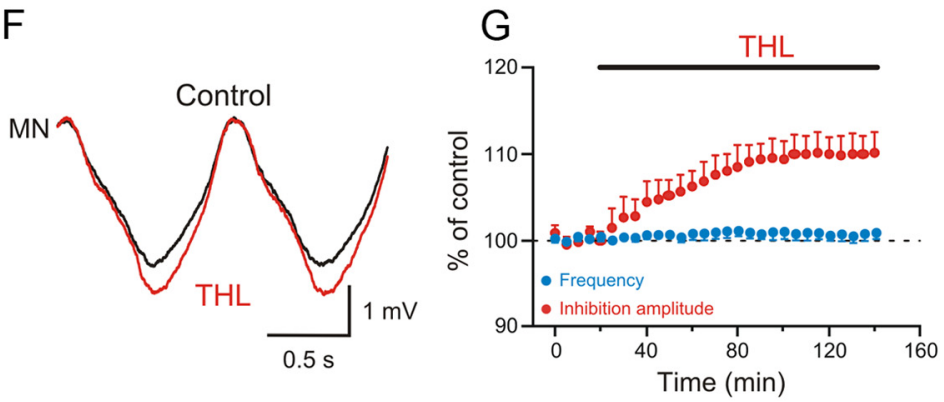

$\mathrm{H}$

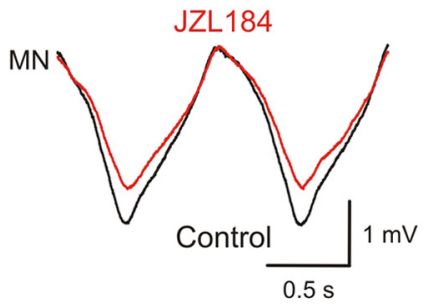

Figure 4. Depression of inhibitory synaptic transmission by the endocannabinoid 2-AG. $A$, The recoding chamber was divided into two pools with a agar barrier, and locomotor activity was induced by NMDA in the rostral part while locomotor-driven excitation was recorded in a MN in the caudal pool, which was perfused with normal saline solution containing kynurenic acid (KynA) to block excitation. $\boldsymbol{B}$, The recorded MN received inhibitory inputs occurring in phase with the contralateral ventral root burst. C, Application of 2-AG to the caudal pool decreased the amplitude of inhibitory synaptic inputreceived by the MN. D, Averaged inhibitory synaptic inputreceived by the MN showing a decrease in amplitude induced by 2-AG. $\boldsymbol{E}$, Plot showing average data from different experiments showing the time course of the change in the amplitude of the inhibition in the caudal pool (red) and the locomotor burs frequency in the rostral pool (blue) before and during 2-AG application. $\boldsymbol{F}$, Average inhibitory synaptic input in control and in THL. G, Plot of averaged data from different experiments showing the time course of the change in the amplitude of the inhibition in the caudal pool (red) and the locomotor burst frequency in the rostral pool (blue) in control and in THL. H, Application of JZL184 in the caudal pool decreased the amplitude of the inhibitory input received by the MN. I, Plot of averaged data from different experiments showing the change in the amplitude of the inhibition in the caudal pool (red) and the locomotor burst frequency in the rostral pool (blue) in control and in JZL184.

showed that endocannabinoids and NO are recruited by metabotropic glutamate receptor 1 (mGluR1) activation and act synergistically to modulate the locomotor activity and synaptic transmission (Kyriakatos and El Manira, 2007; Kyriakatos et al., 2009). We therefore tested whether $\mathrm{NO}$ is involved in reversing the polarity of endocannabinoid modulation. For this, we performed experiments using the split-bath chamber to monitor the modulation of excitatory (with strychnine in the caudal pool) and inhibitory (with kynurenic acid in the caudal pool) synaptic transmission by 2-AG during locomotion in preparations preincubated with carboxy-PTIO (150 $\mu \mathrm{m} ; 1 \mathrm{~h}$ in the caudal pool), which is preferentially an extracellular NO scavenger (Griffiths et al., 2003). Preincubation with carboxy-PTIO completely blocked 2-AG-induced increase in excitatory synaptic transmission $(p<$ $0.01 ; n=7$; Fig. $5 A-D$; in Fig. $5 D$ : carboxy-PTIO, black, and control, gray), indicating that the potentiation of the excitatory drive during locomotion by endocannabinoids is entirely dependent on NO signaling. In contrast, the 2-AGinduced depression of inhibitory synaptic transmission arising from commissural interneurons was not affected by carboxyPTIO (Fig. 6A,B). The decrease in the amplitude of the inhibitory synaptic potentials by $2-A G$ in preparations preincubated with carboxy-PTIO (black) was not significantly different from that obtained in control (gray) experiments $(p>0.05 ; n=6$; Fig. $6 C, D)$. These results show that $\mathrm{NO}$ acts to switch the sign of cannabinoid-induced modulation of synaptic transmission, hence resulting in a more pronounced shift in the balance between excitation and inhibition within the locomotor circuit.

The question that stems from the above results is whether the potentiation of the burst frequency induced by endocannabinoids in the whole locomotor circuit is a direct consequence of the differential modulation of excitatory and inhibitory synaptic transmission. To test for this, the effect of 2-AG on the locomotor burst frequency and membrane potential oscillation was tested in the presence of carboxy-PTIO $(150 \mu \mathrm{M})$. Application of $2-A G$ in preparations preincubated with carboxy-PTIO could still induce a potentiation of the locomotor burst frequency; however, the increase was significantly smaller than that in control [carboxyPTIO (blue), $108.4 \pm 2.5 \% ; n=6$; control (gray), $117.9 \pm 3.9 \% ; p<0.01 ; n=6$; Fig. $7 A, B]$. This is despite the fact that the decrease in the amplitude of the membrane 
potential oscillations was significantly more pronounced in carboxy-PTIO by $23.4 \pm$ $3.7 \%$ (red) than that without carboxy-PTIO by $16.3 \pm 4.7 \%$ (gray) $(p<0.01 ; n=6$; Fig. $7 C, D)$. These results thus indicate that the interplay between cannabinoid and NO signaling underlies a concomitant enhancement of the excitatory drive and a decrease of midcycle inhibition, which produces a more pronounced shift in favor of increased excitability in the spinal locomotor circuit and hence a potentiation of the burst frequency.

\section{Discussion \\ Embedded endocannabinoid modulation and its gating by nitric oxide}

In this study, we first show that 2-AG is responsible for the endocannabinoidembedded modulation of the locomotor frequency and synaptic transmission by interfering with its synthesis and degradation. Blocking the synthesis or degradation of 2-AG produced opposite effects on the locomotor frequency as well as on the underlying on-cycle excitation and midcycle inhibition. In addition, we show that NO switches the sign of endocannabinoid modulation of synaptic transmission within the locomotor CPG. The NO gating enables endocannabinoids to simultaneously potentiate excitation and depress inhibition (Fig. $8)$. Our results indicate that the endocannabinoid 2-AG is mobilized from network neurons during locomotor activity and plays a significant role, in synergy with $\mathrm{NO}$, in setting the baseline burst frequency by adjusting the excitability balance within the locomotor CPG. Thus, although the final locomotor output is generated by activation of ionotropic receptors, it is also heavily dependent on the embedded modulation by endocannabinoids, which plays a buffering role to set the baseline operation of the locomotor CPG.

\section{Identification of the endocannabinoid mobilized in the spinal locomotor CPGs}

The two main endocannabinoids are 2-AG and anandamide, and they are synthesized and metabolized by separate pathways (Sugiura et al., 2002, 2006; Piomelli, 2003; Di Marzo, 2008). However, the identity of the endocannabinoid regulating the locomotor activity has until now been unclear. In the dorsal horn, anatomical evidence using electron microscopy showed postsynaptic localization of DGL- $\alpha$ at nociceptive synapses formed by primary afferents, and a presynaptic positioning of $\mathrm{CB}_{1}$ Rs on excitatory axon terminals (Nyilas et al., 2009; Pernía-Andrade et al., 2009). DGL- $\alpha$ in postsynaptic elements

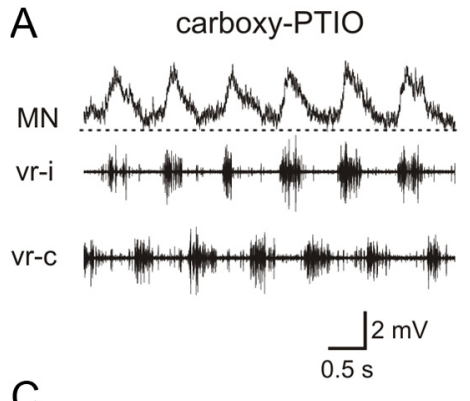

B carboxy-PTIO + 2-AG
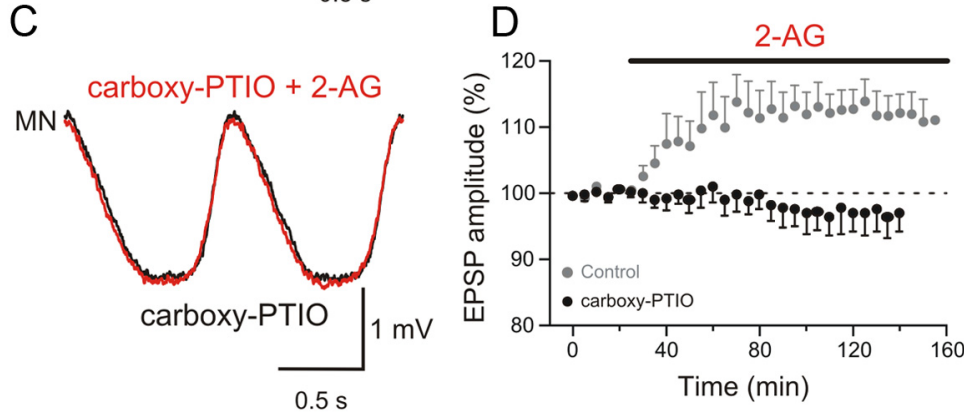

Figure 5. Nitric oxide is required for the potentiation of the excitation by the endocannabinoid 2-AG. A, Excitatory synaptic input received by a motoneuron was recorded in a split-bath chamber with strychnine in the caudal pool (Fig. 3). The caudal pool was preincubated with the NO scavenger carboxy-PTIO for 1 h. B, Application of 2-AG in the caudal pool in the presence of carboxy-PTIO failed to potentiate the amplitude of the excitatory input received by the MN. $C$, Average excitatory synaptic input recorded in a MN preincubated with carboxy-PTIO before and during 2-AG application. D, Plot of averaged data from different experiments showing the time course of the change in the amplitude of the excitation in the presence of carboxy-PTIO before and during 2-AG application (black data points). The gray data points show the change in the excitation induced by 2-AG in the absence of carboxy-PTIO (same data as in Fig. 3E).

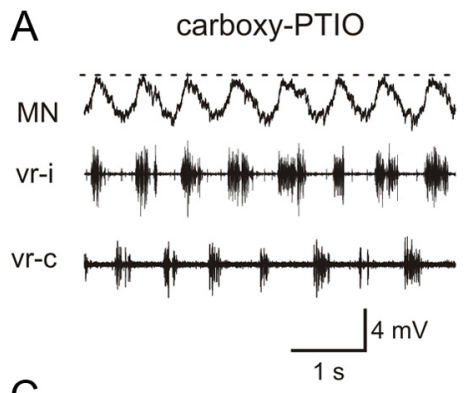

B carboxy-PTIO + 2-AG
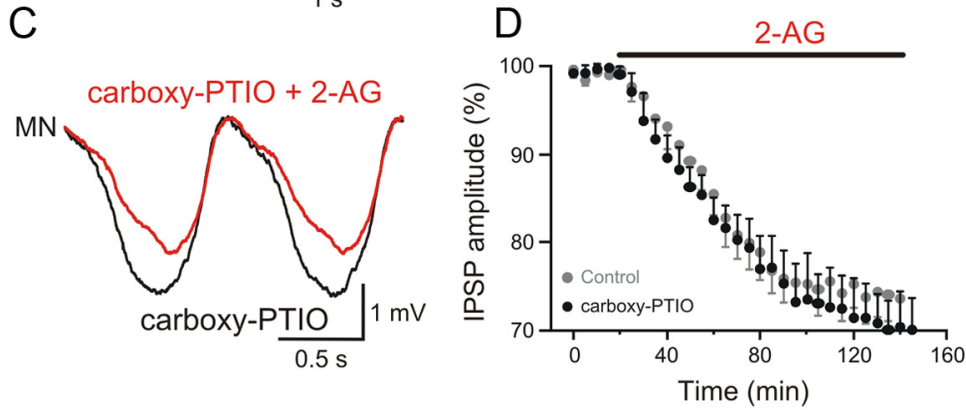

Figure 6. Depression of inhibition by the endocannabinoid 2-AG is not dependent on nitric oxide. $A$, Inhibitory synaptic input received by a motoneuron was recorded in a split-bath chamber with kynurenic acid in the caudal pool (Fig. 4). The caudal pool was preincubated with the NO scavenger carboxy-PTIO for 1 h. B, Application of 2-AG in the caudal pool in the presence of carboxy-PTIO was still able to depress the amplitude of the inhibitory input received by the MN. C, Average inhibitory synaptic input recorded in a MN preincubated with Carboxy-PTIO before and during 2-AG application. D, Plot of averaged data from different experiments showing the time course of the change in the amplitude of the inhibition in the presence of carboxy-PTIO before and during 2-AG application (black data points). The gray data points show the change in the inhibition induced by 2-AG in the absence of carboxy-PTIO (same data as in Fig. 4E).

receiving nociceptive input was colocalized with mGluR5, whose activation induces 2-AG biosynthesis (Nyilas et al., 2009). Blocking of the DGL, which hydrolyzes DAG into 2-AG, decreased the frequency of the locomotor rhythm by depressing the excitation and enhancing the inhibition. This indicates that there is a con- 
A carboxy-PTIO

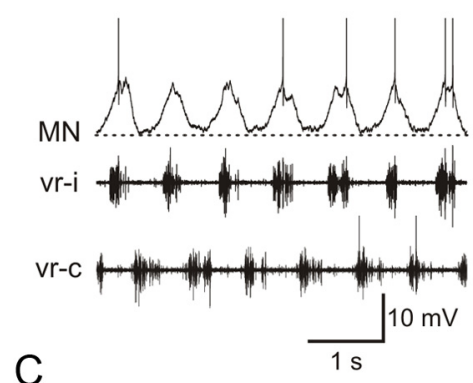

C carboxy-PTIO + 2-AG
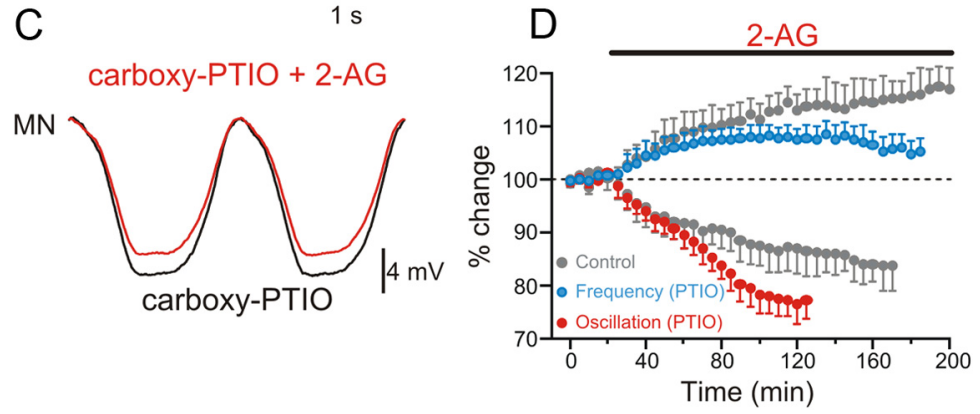

Figure 7. Nitric oxide contributes to the increase in the locomotor burst frequency by the endocannabinoid 2-AG. A, Locomotor rhythm was induced by NMDA in the presence of the NO scavenger carboxy-PTIO and was recorded in opposing ventral roots. Intracellular recording from a MN that received on-cycle excitation in phase with the ipsilateral ventral root (vr-i) activity that alternated with midcycle inhibition occurring in phase with the contralateral ventral root (vr-c) burst. B, Application of the endocannabinoid 2-AG (2 $\mu \mathrm{m})$ in the presence of carboxy-PTIO was still able to increase the burst frequency that was associated with a decrease in the oscillation amplitude. $C$, Averaged synaptic inputs received by the $\mathrm{MN}$ showing a decrease in the amplitude of the oscillation by 2 -AG. In these recordings, the action potentials were filtered out using a low-pass filter, normalized, and aligned in relation to the membrane potential of the MN. $\boldsymbol{D}$, Averaged data from all the experiments showing the time course of the effect of 2-AG on synaptic transmission and the locomotor burst frequency in the presence of carboxy-PTIO. The decrease of the oscillation amplitude was more pronounced (red), while the potentiation of the locomotor burst frequency was attenuated (blue) in preparations preincubated with carboxy-PTIO compared with controls (gray; same data as in Fig. 1D).
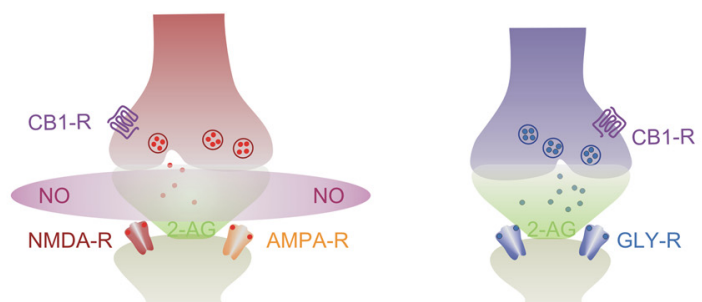

Postsynaptic motoneuron
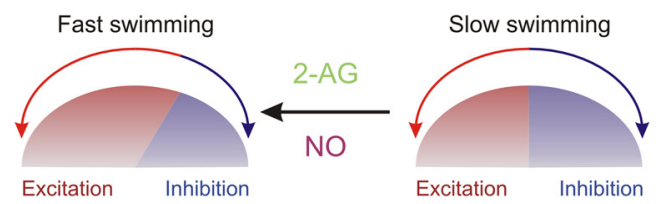

Figure 8. Summary of the interplay between the endocannabinoid 2-AG and nitric oxide. The intimate interplay between endocannabinoid and MN induces a switch in the balance between excitation and inhibition within the locomotor circuit and thus mediates an increase in swimming frequency.

tinuous synthesis of 2-AG within the locomotor networks that helps to maintain the baseline level of activity. In addition, blocking MGL, the enzyme that degrades 2-AG, increased the locomotor burst frequency by potentiating the excitation and depressing the inhibition. Indeed, by affecting the synthesizing (DGL) and degrading (MGL) enzymes, we produce opposite effects on the locomotor rhythm and the underlying on-cycle excitation and midcycle inhibition. These results argue that 2-AG is released in the spinal locomotor CPG that is continuously synthesized and helps modulating the baseline excitability underlying the locomotor output. Our results do not exclude the possibility that, in addition to the on-demand release of endocannabinoids, there is also a constitutive synthesis and mobilization of endocannabinoids independent of the activity of the spinal locomotor network.

In keeping with these results, we have previously shown that activation of mGluR1 induces short- and long-term potentiation of the locomotor activity (Krieger et al., 2000; El Manira et al., 2002, 2010; Kyriakatos and El Manira, 2007). This receptor is coupled to the G-protein Gq/11, which activates phospholipase $\mathrm{C}$ (Kettunen et al., 2002, 2003; Nanou et al., 2009; Nanou and El Manira, 2010); this signaling pathway yields to accumulation of DAG that in turn is hydrolyzed by DGL into 2-AG (Safo and Regehr, 2005; Chevaleyre et al., 2006; Heifets and Castillo, 2009; Kano et al., 2009; Tanimura et al., 2010). The long-term potentiation of the locomotor burst frequency was completely dependent on activation of $\mathrm{CB}_{1}$ Rs because it was blocked by the antagonist AM251 (Kyriakatos and El Manira, 2007). Evidence exists to suggest that $2-A G$ or anandamide represents the endocannabinoid released in many regions in the CNS. A direct activation of $\mathrm{CB}_{1} \mathrm{R}$ by endocannabinoids, be it 2-AG or anandamide, has been shown to produce exclusively depression of both excitatory and inhibitory synaptic transmission in many regions of the CNS (Alger, 2002; Wilson and Nicoll, 2002; Freund et al., 2003; Chevaleyre et al., 2006; Katona and Freund, 2008; Kreitzer and Malenka, 2008; Lovinger, 2008; Heifets and Castillo, 2009; Kano et al., 2009). In hippocampus, cerebellum, and hypothalamus, the depression of synaptic transmission has been suggested to involve an interaction between $\mathrm{NO}$ and endocannabinoid signaling (Safo and Regehr, 2005; Makara et al., 2007; Crosby et al., 2011). In goldfish, however, it has been shown that endocannabinoids indirectly potentiate excitatory synaptic transmission via release of dopamine (Cachope et al., 2007).

\section{Nitric oxide and endocannabinoids in the spinal locomotor networks}

Several classes of neurons are known to be involved in motor control and sensorimotor integration in the lamprey spinal cord. These include gray matter neurons (motoneurons and interneurons), proprioceptive edge cells, which express NO synthase (NOS) and are therefore potential intrinsic sources of NO (Kyriakatos et al., 2009). We previously showed that the NOS inhibitor L-NAME and the NO scavenger carboxyPTIO decrease the locomotor frequency, and the latter also blocked NO donor-induced modulation. These results indicate that NO mediates a positive-feedback control of the network 
during swimming to maintain the rhythm at a high frequency (Kyriakatos et al., 2009). In Xenopus tadpoles, NO exerts mainly inhibitory actions by selectively enhancing GABAergic descending inhibition from mid-hindbrain reticulospinal interneurons and by enhancing glycinergic inhibition during swimming (McLean and Sillar, 2002). This latter effect involves metamodulation of the effects of noradrenaline (McLean and Sillar, 2004). Endocannabinoids are mobilized within the lamprey locomotor network and modulate inhibitory synaptic transmission and locomotor frequency (Kettunen et al., 2005; El Manira et al., 2008; El Manira and Kyriakatos, 2010). Endocannabinoid and NO signaling were thought to act only in parallel to modulate the activity of the spinal locomotor network. Our results now show that endocannabinoids and NO signaling are in addition intimately coupled to mediate selective modulation of excitatory and inhibitory synaptic transmission and regulate the locomotor network output.

\section{Nitric oxide switches the sign of endocannabinoid modulation of synaptic transmission}

An important, previously unknown interaction between endocannabinoids and $\mathrm{NO}$ is in the selective potentiation of excitatory synaptic transmission. This potentiation, combined with a direct depression of the inhibition by activation of presynaptic $C_{1} R$, adjusts the excitability balance in favor of increased excitatory transmission and hence a potentiation of the locomotor burst frequency (Fig. 8). The interaction between NO and endocannabinoids is restricted to excitatory synaptic transmission and was blocked by the membrane-impermeable scavenger carboxyPTIO (Griffiths et al., 2003), indicating that NO diffuses outside the neurons where it is produced. This leads to a decrease of inhibitory synaptic transmission by activation of presynaptic $\mathrm{CB}_{1}$ Rs. One prevalent mechanism of $\mathrm{NO}$ synthesis is via $\mathrm{Ca}^{2+}$ influx through NMDA receptors, leading to activation of NOS, which is commonly tethered to NMDA receptors (Brenman and Bredt, 1997; Prast and Philippu, 2001; Garthwaite, 2008). We previously showed that inhibition of NOS by L-NAME affects the locomotor frequency in the lamprey spinal cord (Kyriakatos et al., 2009) and blocked the endocannabinoid-dependent potentiation of the burst frequency induced by mGluR1 (Kyriakatos and El Manira, 2007). The NO-generated and the mobilized endocannabinoids interact to produce potentiation of excitatory synaptic transmission. It thus seems that $\mathrm{NO}$ acts as a metamodulator to determine the polarity of the endocannabinoid-mediated plasticity of excitation. Ultimately, the balance between excitatory and inhibitory synaptic transmission determines the speed and force of the locomotor movements produced by the spinal locomotor CPG. The gating of the sign of endocannabinoid-mediated synaptic plasticity by NO represents a novel mechanism by which the excitability balance is appropriately set within the locomotor CPG to allow for a more flexible execution of motor behavior.

\section{References}

Abbott LF, Regehr WG (2004) Synaptic computation. Nature 431:796-803. Albin RL, Young AB, Penney JB (1989) The functional anatomy of basal ganglia disorders. Trends Neurosci 12:366-375.

Alger BE (2002) Retrograde signaling in the regulation of synaptic transmission: focus on endocannabinoids. Prog Neurobiol 68:247-286.

Bisogno T, Howell F, Williams G, Minassi A, Cascio MG, Ligresti A, Matias I, Schiano-Moriello A, Paul P, Williams EJ, Gangadharan U, Hobbs C, Di Marzo V, Doherty P (2003) Cloning of the first snl-DAG lipases points to the spatial and temporal regulation of endocannabinoid signaling in the brain. J Cell Biol 163:463-468.

Brenman JE, Bredt DS (1997) Synaptic signaling by nitric oxide. Curr Opin Neurobiol 7:374-378.
Büschges A, Scholz H, El Manira A (2011) New moves in motor control. Curr Biol 21:R513-R524.

Cachope R, Mackie K, Triller A, O’Brien J, Pereda AE (2007) Potentiation of electrical and chemical synaptic transmission mediated by endocannabinoids. Neuron 56:1034-1047.

Chevaleyre V, Takahashi KA, Castillo PE (2006) Endocannabinoidmediated synaptic plasticity in the CNS. Annu Rev Neurosci 29:37-76.

Crosby KM, Inoue W, Pittman QJ, Bains JS (2011) Endocannabinoids gate state-dependent plasticity of synaptic inhibition in feeding circuits. Neuron 71:529-541.

Dale N (1986) Excitatory synaptic drive for swimming mediated by amino acid receptors in the lamprey. J Neurosci 6:2662-2675.

Destexhe A, Marder E (2004) Plasticity in single neuron and circuit computations. Nature 431:789-795.

Di Marzo V (2008) Endocannabinoids: synthesis and degradation. Rev Physiol Biochem Pharmacol 160:1-24.

El Manira A, Kyriakatos A (2010) The role of endocannabinoid signaling in motor control. Physiology (Bethesda) 25:230-238.

El Manira A, Kettunen P, Hess D, Krieger P (2002) Metabotropic glutamate receptors provide intrinsic modulation of the lamprey locomotor network. Brain Res Brain Res Rev 40:9-18.

El Manira A, Kyriakatos A, Nanou E, Mahmood R (2008) Endocannabinoid signaling in the spinal locomotor circuitry. Brain Res Rev 57:29-36.

El Manira A, Kyriakatos A, Nanou E (2010) Beyond connectivity of locomotor circuitry-ionic and modulatory mechanisms. Prog Brain Res 187:99-110.

Fetcho JR, McLean DL (2010) Some principles of organization of spinal neurons underlying locomotion in zebrafish and their implications. Ann N Y Acad Sci 1198:94-104.

Freund TF, Katona I, Piomelli D (2003) Role of endogenous cannabinoids in synaptic signaling. Physiol Rev 83:1017-1066.

Garthwaite J (2008) Concepts of neural nitric oxide-mediated transmission. Eur J Neurosci 27:2783-2802.

Goulding M (2009) Circuits controlling vertebrate locomotion: moving in a new direction. Nat Rev Neurosci 10:507-518.

Griffiths C, Wykes V, Bellamy TC, Garthwaite J (2003) A new and simple method for delivering clamped nitric oxide concentrations in the physiological range: application to activation of guanylyl cyclase-coupled nitric oxide receptors. Mol Pharmacol 64:1349-1356.

Grillner S (1975) Locomotion in vertebrates: central mechanisms and reflex interaction. Physiol Rev 55:247-304.

Grillner S, Jessell TM (2009) Measured motion: searching for simplicity in spinal locomotor networks. Curr Opin Neurobiol 19:572-586.

Harris-Warrick RM (2011) Neuromodulation and flexibility in central pattern generator networks. Curr Opin Neurobiol 21:685-692.

Hashimotodani Y, Ohno-Shosaku T, Maejima T, Fukami K, Kano M (2008) Pharmacological evidence for the involvement of diacylglycerol lipase in depolarization-induced endocanabinoid release. Neuropharmacology 54:58-67.

Heifets BD, Castillo PE (2009) Endocannabinoid signaling and long-term synaptic plasticity. Annu Rev Physiol 71:283-306.

Jordan LM, Slawinska U (2011) Chapter 12-Modulation of rhythmic movement: control of coordination. Prog Brain Res 188:181-195.

Kano M, Ohno-Shosaku T, Hashimotodani Y, Uchigashima M, Watanabe M (2009) Endocannabinoid-mediated control of synaptic transmission. Physiol Rev 89:309-380.

Katona I, Freund TF (2008) Endocannabinoid signaling as a synaptic circuit breaker in neurological disease. Nat Med 14:923-930.

Kettunen P, Krieger P, Hess D, El Manira A (2002) Signaling mechanisms of metabotropic glutamate receptor 5 subtype and its endogenous role in a locomotor network. J Neurosci 22:1868-1873.

Kettunen P, Hess D, El Manira A (2003) mGluR1, but not mGluR5, mediates depolarization of spinal cord neurons by blocking a leak current. J Neurophysiol 90:2341-2348.

Kettunen P, Kyriakatos A, Hallén K, El Manira A (2005) Neuromodulation via conditional release of endocannabinoids in the spinal locomotor network. Neuron 45:95-104.

Kiehn O (2006) Locomotor circuits in the mammalian spinal cord. Annu Rev Neurosci 29:279-306.

Kim J, Isokawa M, Ledent C, Alger BE (2002) Activation of muscarinic acetylcholine receptors enhances the release of endogenous cannabinoids in the hippocampus. J Neurosci 22:10182-10191. 
Kreitzer AC, Malenka RC (2007) Endocannabinoid-mediated rescue of striatal LTD and motor deficits in Parkinson's disease models. Nature 445:643-647.

Kreitzer AC, Malenka RC (2008) Striatal plasticity and basal ganglia circuit function. Neuron 60:543-554.

Krieger P, Hellgren-Kotaleski J, Kettunen P, El Manira AJ (2000) Interaction between metabotropic and ionotropic glutamate receptors regulates neuronal network activity. J Neurosci 20:5382-5391.

Kristan WB Jr, Calabrese RL, Friesen WO (2005) Neuronal control of leech behavior. Prog Neurobiol 76:279-327.

Kyriakatos A, El Manira A (2007) Long-term plasticity of the spinal locomotor circuitry mediated by endocannabinoid and nitric oxide signaling. J Neurosci 27:12664-12674.

Kyriakatos A, Molinari M, Mahmood R, Grillner S, Sillar KT, El Manira A (2009) Nitric oxide potentiation of locomotor activity in the spinal cord of the lamprey. J Neurosci 29:13283-13291.

LeBeau FE, El Manira A, Griller S (2005) Tuning the network: modulation of neuronal microcircuits in the spinal cord and hippocampus. Trends Neurosci 28:552-561.

Lovinger DM (2008) Presynaptic modulation by endocannabinoids. Handb Exp Pharmacol 2008:435-477.

Mackie K, Stella N (2006) Cannabinoid receptors and endocannabinoids: evidence for new players. AAPS J 8:E298-E306.

Makara JK, Katona I, Nyíri G, Németh B, Ledent C, Watanabe M, de Vente J, Freund TF, Hájos N (2007) Involvement of nitric oxide in depolarizationinduced suppression of inhibition in hippocampal pyramidal cells during activation of cholinergic receptors. J Neurosci 27:10211-10222.

McLean DL, Sillar KT (2002) Nitric oxide selectively tunes inhibitory synapses to modulate vertebrate locomotion. J Neurosci 22:4175-4184.

McLean DL, Sillar KT (2004) Metamodulation of a spinal locomotor network by nitric oxide. J Neurosci 24:9561-9571.

Miles GB, Sillar KT (2011) Neuromodulation of vertebrate locomotor control networks. Physiology (Bethesda) 26:393-411.

Nanou E, El Manira A (2010) Mechanisms of modulation of AMPA-induced $\mathrm{Na}^{+}$-activated $\mathrm{K}^{+}$current by mGluR1. J Neurophysiol 103:441-445.

Nanou E, Kyriakatos A, Kettunen P, El Manira A (2009) Separate signalling mechanisms underlie mGluR1 modulation of leak channels and NMDA receptors in the network underlying locomotion. J Physiol 587:3001-3008.

Nusbaum MP, Blitz DM, Swensen AM, Wood D, Marder E (2001) The roles of co-transmission in neural network modulation. Trends Neurosci 24:146-154.

Nyilas R, Gregg LC, Mackie K, Watanabe M, Zimmer A, Hohmann AG,
Katona I (2009) Molecular architecture of endocannabinoid signaling at nociceptive synapses mediating analgesia. Eur J Neurosci 29:1964-1978.

Pan B, Wang W, Long JZ, Sun D, Hillard CJ, Cravatt BF, Liu QS (2009) Blockade of 2-arachidonoylglycerol hydrolysis by selective monoacylglycerol lipase inhibitor 4-nitrophenyl 4-(dibenzo[ $d][1,3]$ dioxol-5-yl(hydroxy)methyl)piperidine-1-carboxylate (JZL184) enhances retrograde endocannabinoid signaling. J Pharmacol Exp Ther 331:591-597.

Pernía-Andrade AJ, Kato A, Witschi R, Nyilas R, Katona I, Freund TF, Watanabe M, Filitz J, Koppert W, Schüttler J, Ji G, Neugebauer V, Marsicano G, Lutz B, Vanegas H, Zeilhofer HU (2009) Spinal endocannabinoids and CB1 receptors mediate C-fiber-induced heterosynaptic pain sensitization. Science 325:760-764.

Piomelli D (2003) The molecular logic of endocannabinoid signalling. Nat Rev Neurosci 4:873-884.

Prast H, Philippu A (2001) Nitric oxide as modulator of neuronal function. Prog Neurobiol 64:51-68.

Roberts A, Li WC, Soffe SR (2010) How neurons generate behavior in a hatchling amphibian tadpole: an outline. Front Behav Neurosci 4:16.

Safo PK, Regehr WG (2005) Endocannabinoids control the induction of cerebellar LTD. Neuron 48:647-659.

Sillar KT, Combes D, Ramanathan S, Molinari M, Simmers J (2008) Neuromodulation and developmental plasticity in the locomotor system of anuran amphibians during metamorphosis. Brain Res Rev 57:94-102.

Sugiura T, Kobayashi Y, Oka S, Waku K (2002) Biosynthesis and degradation of anandamide and 2-arachidonoylglycerol and their possible physiological significance. Prostaglandins Leukot Essent Fatty Acids 66:173-192.

Sugiura T, Kishimoto S, Oka S, Gokoh M (2006) Biochemistry, pharmacology and physiology of 2-arachidonoylglycerol, an endogenous cannabinoid receptor ligand. Prog Lipid Res 45:405-446.

Surmeier DJ, Ding J, Day M, Wang Z, Shen W (2007) D1 and D2 dopaminereceptor modulation of striatal glutamatergic signaling in striatal medium spiny neurons. Trends Neurosci 30:228-235.

Tanimura A, Yamazaki M, Hashimotodani Y, Uchigashima M, Kawata S, Abe M, Kita Y, Hashimoto K, Shimizu T, Watanabe M, Sakimura K, Kano M (2010) The endocannabinoid 2-arachidonoylglycerol produced by diacylglycerol lipase alpha mediates retrograde suppression of synaptic transmission. Neuron 65:320-327.

Toledo-Rodriguez M, El Manira A, Wallén P, Svirskis G, Hounsgaard J (2005) Cellular signalling properties in microcircuits. Trends Neurosci 28:534-540.

Wilson RI, Nicoll RA (2002) Endocannabinoid signaling in the brain. Science 296:678-682. 